\title{
Simultaneous determination of furosemide and spironolactone in pharmaceutical formulations by spectrophotometric method using principal component regression
}

\author{
S. S. Israt ${ }^{1}$, M. N. Uddin ${ }^{2}$, R. A. Jahan ${ }^{3}$, and M. M. Karim ${ }^{1 *}$ \\ ${ }^{I}$ Department of Applied Chemistry and Chemical Engineering, University of Dhaka, Dhaka-1000, Bangladesh \\ ${ }^{2}$ Bangladesh Council of Scientific \& Industrial Research (BCSIR), Dhaka-1205, Bangladesh \\ ${ }^{3}$ Centre for Advanced Research in Sciences, University of Dhaka, Dhaka-1000, Bangladesh
}

\begin{abstract}
The combination of furosemide (furo) and spironolactone (spiro) is very effective in the treatment of heart failure. In that case, maintaining good quality of these drugs in commercial tablets is must. Therefore, a simple, economical, precise and accurate method, i.e; chemometric assisted UV spectroscopy, for simultaneous determination of furosemide and spironolactone in combined dosage form has been developed. In this study, principal component regression (PCR) has been reported for this purpose. A calibration set of 36 mixture solutions containing furosemide and spironolactone in methanol in the concentration range of 2.0-12.0 $\mu \mathrm{g} / \mathrm{ml}$ and 5.0-30.0 $\mu \mathrm{g} / \mathrm{ml}$ respectively has been prepared by means of an orthogonal experimental design. The absorbance data for the concentration set have been obtained by direct measurement in UV spectrophotometer at 101 wavelength points in the spectral region of 200-300 nm for the zero order spectra. The chemometric technique is also successfully applied to available pharmaceutical formulations, tablets, with no interference from excipients. The analytical performances of principal component regression are characterized by relative prediction errors and recoveries (\%). The good recoveries obtained in this case proved that the proposed chemometric technique could be applied efficiently in the quality control of the studied drugs simultaneously in their mixture as well as in the commercial dosage form with satisfactory precision and accuracy as alternative analysis tools.
\end{abstract}

Keywords: Furosemide; Spironolactone; Chemometric; Principal component regression; UV spectroscopy

\section{Introduction}

Spironolactone (spiro) called a potassium-sparing diuretic and furosemide (furo), a loop diuretic are widely used and frequently co-formulated in pharmaceutical preparations. Diuretics function in our kidneys. They work by causing the kidneys to increase the amount of salts, such as potassium and sodium, which are filtered out of the blood and into the urine. When these salts are filtered out of the blood by the kidneys, they draw water alongside them. As diuretics increase the removal of salts from the blood, they also cause more water to be drawn out from the blood into the urine. Removing water from the blood decreases the volume of fluid circulating through the blood vessels. This subsequently decreases the pressure within the blood vessels. Diuretics such as spironolactone and furosemide can therefore be used to lower high blood pressure.
Loop diuretics like furosemide are strong diuretics that cause lots of potassium to be filtered out of the blood. This can sometimes cause the level of potassium in the blood to fall too low. A low potassium level blood is called hypokalaemia and can sometimes be dangerous, particularly for people with heart disease or liver disease. Spironolactone works by blocking the actions of a hormone in the body called aldosterone. It inhibits the effect of aldosterone by competing for intracellular aldosterone receptors in the distal tubule cells. This increases the excretion of water and sodium, while decreasing the excretion of potassium. Spironolactone is also used in heart failure, where the pumping mechanism of the heart is less effective. It is used to relieve the symptoms of heart failure, such as the shortness of breath seen with fluid on the lungs. It can also be used to remove excess fluid that can accumulate in the body in people with cirrhosis of the

*Corresponding author e-mail: moinulcd@yahoo.com 
liver. This fluid may accumulate in the abdomen (ascites) or in the legs (oedema).

Spironolactone has a fairly slow onset of action, taking several days to develop; similarly, the effect diminishes slowly (Ram et al., 2012). In clinical practice, spironolactone is frequently given in combination with loop diuretic like furosemide. The advantages of this combination over spironolactone alone include a greater natriuretic potency, earlier onset of dieresis and fewer tendencies to induce hyperkalemia (Ginès et al., 2010). Therefore, the spironolactone-furosemide combination is widely prescribed.

Quality control of dosage form preparations of drug needs reliable and quick analytical methods. UV/vis spectrophotometry is by far the instrumental technique of choice in industrial laboratories, owing mainly to its rapidness, simplicity, and sensitivity and often requiring low-cost equipment. Simultaneous determination of pharmaceuticals containing multi-active absorbing ingredients is difficult to perform by classical spectrophotometric technique due to spectral overlap.

Recently, multivariate calibration methods appear to be the proper techniques that show the best performance for complex mixture resolution (Vigneau et al., 1997; Massart et al., 1998; Lavine 2000; Haaland et al., 2000; Fearn, 2001; Brereton, 2003; Geladi, 2003; Ragno et al., 2004). Multivariate calibration is an effective calibration method in which the chemical information (absorbance, emission, transmission, etc.) of a set of standard mixtures recorded at different variables (wavenumbers) are related to the concentration of the chemical compounds present in the mixtures (Martens and Naes, 1991). The popular calibration way used in chemical analysis is the univariate calibration in which the chemical information of a set of solution recorded at one variable (i.e. wavenumber) is related to the solute concentration in the solution. Univariate calibration has no fault-detection capabilities. The presence of interference results in the overestimation of the concentration of the analyte of interest. In addition, the analyst will not be aware of the problem. Using univariate measurement schemes, there are two ways to avoid interferents problems-physically separating the analytes of interest from all interferents and /or using selective measurements. Although both approaches are often acceptable and successful, considerable effort can be expended in developing the separations and/ or measurements. Improved precision and accuracy over univariate methods can often be realized because of the multivariate advantage. The most applied multivariate calibration tools are classical least squares (CLS), inverse least squares (ILS), principal component regression (PCR), and partial least squares (PLS) and support vector machine (SVM). The diagnostics are excellent in both the calibration and prediction phasesand used as satisfactory solution in most cases, where a linear relationship is present between the analytical signal and the property to be determined (Beebe et al., 1998). Among them principal component regression (PCR) is a regression technique based on principal component analysis (PCA). PCR does not require wavelength selection. Any number can be used; usually the whole spectrum, or large regions. Larger number of wavelengths gives averaging effect, making model less susceptible to spectral noise.

One major use of PCR lies in overcoming the multicollinearity problem which arises when two or more of the explanatory variables are close to being collinear. PCR can aptly deal with such situations by excluding some of the lowvariance principal components in the regression step. In addition, by usuing regressing on only a subset of all the principal components, PCR can result in dimension reduction through substantially lowering the effective number of parameters characterizing the underlying model. This can be particularly useful in settings with high-dimensional covariates. Also, through appropriate selection of the principal components to be used for regression, PCR can lead to efficient prediction of the outcome based on the assumed model. PCR offers an analytical chemist several advantages. One can separate and retain PCs that describe relevant information and discard PCs that contain noise, thereby eliminating sources of random error. PCs that describe relevant information should have larger projections because they describe more of the collected data set than those that describe noise, which should be a small percentage of the overall measured signal. There are numerous ways to decide how many PCs to keep, but all rely on the same basic assumption that PCs that describe relevant information will describe more of the collected data than PCs that describe only noise. Second, the size of a data matrix is drastically reduced. An entire spectrum can be replaced by its distance (or projection) along a few PCs (Keithley et al., 2009)

In spite of their wide use and the availability of many analytical methods only a few scattered reports are available on either individual or in a combination of spironolactone and furosemide including simultaneous quantification of spironolactone with hydroflumethiazide and spironolactone 
with furosemide in combination formulations by ultraviolet (UV) absorption and UV derivative spectrophotometry, respectively (Parimoo et al., 1995; Salem et al., 1991); spectrophotometric determination using complex formation with $\mathrm{Cu}$ (II) (Gole Cure, 2006); liquid chromatography for biological samples (Shaikh, 1995; Overdiek et al., 1985, Basavaiah, and Chandrashekar, 2005), first order derivative spectrophotometric method (Patel and Solanki, 2012) and thin-layer chromatographic determination (Wesley-Hadzija and Mattocks, 1982). None of these methods entails the use of multivariate procedures such as chemometrics, in spite of their recognized high-resolution potential that has been used advantageously for the elimination of interferences and the resolution of overlapped signals. Furthermore, described in this paper is a rapid, precise and accurate procedure (without loss of prediction accuracy) for the simultaneous determination of furosemide and spironolactone in commercial combined tablet preparations, based on the joint use of the PCR and UV-visible spectrophotometric data. As far as the authors know this is the first application of PCR in the direct spectrophotometric determination of diuretics in pharmaceutical formulations.

\section{Materials and methods}

\section{Chemicals}

Active pharmaceutical ingredients such as furosemide and spironolactone were kindly donated by Acme Laboratories Limited (Bangladesh) and were used without further purification. Five different commercial pharmaceutical preparations like Edeloss by Incepta Pharmaceuticals, Edenil ${ }^{\circledR} 20$ by Eskayef Bangladesh Ltd, Frulac ${ }^{\circledR}$ by Orion Pharma Ltd, Fusid ${ }^{\circledR}$ Plus by Square Pharmaceuticals Ltd, and Resitone ${ }^{\circledR}$ by Beximco Pharma, were purchased from the local resources and analyzed by the proposed chemometric method. Their declared content in the label was as follows: furosemide $20 \mathrm{mg}$ and spironolactone $50 \mathrm{mg}$ in each tablet.

All chemicals used throughout the work were of spectroscopic grade. After assessing the solubility of drugs in different solvents methanol, $(\mathrm{MeOH})$ has been selected as common solvent for developing spectral characteristics. Methanol was purchased from Sigma-Aldrich Chemie Gmbh (Munich, Germany). The water utilized in all studies was double-distilled and deionized.

\section{Apparatus and software}

Shimadzu UV-visible spectrometer (Model: UV-1800, Shimadzu, Kyoto, Japan), equipped with $1 \mathrm{~cm}$ matched quartz cells was used for spectrometric measurements. Experimental design was performed by using the software SPSS (version 22.0). CAMO the UNSCRAMBLER 10.3 has been used for PCR.

\section{Procedure}

One-component calibration: About $10 \mathrm{mg}$ of each furosemide and spironolactone working standards were accurately weighed out, transferred to two separate $100 \mathrm{ml}$ volumetric flask and dissolved in methanol. The volume was made up to the mark to obtain the final concentration of $100 \mu \mathrm{g} / \mathrm{ml}$ for each of the two drugs. The above standard stock solutions of furosemide and spironolactone were used for constructing the individual calibration lines, the absorbances were measured at 272, and $235 \mathrm{~nm}$ against the blank for furosemide and spironolactone, respectively. The linear dynamic ranges determined by regressing absorbances at the corresponding $\lambda_{\max }$ vs. concentrations were 2.0$12.0 \mu \mathrm{g} / \mathrm{ml}$ for furosemide and $5.0-30.0 \mu \mathrm{g} / \mathrm{ml}$ for spironolactone.

\section{Calibration mixtures}

Six different concentrations (ppm) within the linear range of furosemide $(2.0,4.0,6.0,8.0,10.0$ and 12.0) and spironolactone $(5.0,10.0,15.0,20.0,25.0$ and 30.0) were considered to make mixture solution of standard sample by following orthogonal experimental design (OED). Thus 36 solutions were prepared where correlation of concentrations of furosemide and spironolactone were zero. Table I shows the compositions of all calibrations mixtures.

To develop the chemometric calibration, thirty six preparations (calibration set) of standard solutions containing mixture of furosemide and spironolactone were prepared as follows: Appropriate volume of standard stock solutions of furosemide and spironolactone in the concentration ranges between $2.0-12.0 \mu \mathrm{g} / \mathrm{ml}$ for furosemide and $5.0-30.0 \mu \mathrm{g} / \mathrm{ml}$ for spironolactone were added in separate $10 \mathrm{ml}$ flasks. Then the solutions were diluted to $10 \mathrm{ml}$ with methanol. After that a vortex mixer was used to mix the solutions of furosemide and spironolactone uniformly with the solvent for each of the thirty six preparations.

UV spectra of the mixtures were recorded in the wavelength 
range of 200-300 $\mathrm{nm}$ versus a solvent blank and digitized absorbance was sampled at $1 \mathrm{~nm}$ intervals. 101 absorbance data were obtained from the spectrum in the wavelength range of 200-300 $\mathrm{nm}$ for each sample.

Table I. Mixing proportion of furosemide and spironolactone by OED

\begin{tabular}{|c|c|c|}
\hline Sample ID & Furosemide (ppm) & Spironolactone (ppm) \\
\hline 1. & 12 & 30 \\
\hline 2. & 10 & 5 \\
\hline 3. & 10 & 10 \\
\hline 4. & 2 & 20 \\
\hline 5. & 2 & 25 \\
\hline 6. & 10 & 30 \\
\hline 7. & 12 & 15 \\
\hline 8. & 8 & 25 \\
\hline 9. & 6 & 10 \\
\hline 10. & 10 & 20 \\
\hline 11. & 8 & 20 \\
\hline 12. & 4 & 25 \\
\hline 13. & 8 & 30 \\
\hline 14. & 8 & 10 \\
\hline 15. & 6 & 20 \\
\hline 16. & 10 & 15 \\
\hline 17. & 12 & 10 \\
\hline 18. & 8 & 5 \\
\hline 19. & 12 & 5 \\
\hline 20. & 4 & 30 \\
\hline 21. & 6 & 15 \\
\hline 22. & 2 & 5 \\
\hline 23. & 4 & 5 \\
\hline 24. & 4 & 20 \\
\hline 25. & 6 & 25 \\
\hline 26. & 4 & 10 \\
\hline 27. & 2 & 30 \\
\hline 28. & 6 & 30 \\
\hline 29. & 2 & 15 \\
\hline 30. & 8 & 15 \\
\hline 31. & 12 & 20 \\
\hline 32. & 2 & 10 \\
\hline 33. & 10 & 25 \\
\hline 34. & 6 & 5 \\
\hline 35. & 4 & 15 \\
\hline 36. & 12 & 25 \\
\hline
\end{tabular}

\section{Real sample preparations}

Ten tablets were weighed and average weight was calculated. The tablets were ground to finely powdered in a mortar. A portion of the powder equivalent to the average tablet weight was accurately weighed, transferred into a $100 \mathrm{ml}$ volumetric flask, suspended in methanol and then the flask was half filled with the same solvent. The suspension of the flask was mechanically shaken for thirty minutes, filtered into a $100 \mathrm{ml}$ volumetric flask through a filter paper and made up to the mark. The final tablet solution was further diluted quantitatively with methanol to obtain the working concentration range in a $10 \mathrm{ml}$ volumetric flask for UV measurements. The absorption spectra of these drugs were recorded by using the procedure mentioned above for PCR application.

\section{Results and discussion \\ Spectral characteristics of the analytes}

The electronic absorption spectra of pure furosemide (8ppm) and spironolactone (10ppm), as well as the spectrum of a mixture (by weight) of both drugs in $\mathrm{MeOH}$ over the wavelength range 200-300 $\mathrm{nm}$ are shown in Fig. 1. Furosemide displays an absorption maximum at $272 \mathrm{~nm}$ and spironolactone exhibits a maximum at $235 \mathrm{~nm}$. Data analysis anticipated that the severe spectral overlapping observed coupled to the high spiro/furo ratio present in the pharmaceutical preparations could seriously hinder the resolution of the mixture by conventional spectrophotometry. Simultaneous determination of furosemide and spironolactone by evaluating the absorbance readings at several different wavelengths also proved to be impractical in the present case, mainly due to the presence of small amounts of declared absorbing excipients, which are different in the various commercial brands subjected to analysis. In addition, it also precluded the use of derivative spectrophotometry. Thus, PCR is sought as an alternative, since this method can handle severe spectral overlap and a high analyte concentration ratio, and knowledge of the spectra of all the absorbing species is not absolutely necessary.

PCR analysis and evaluation

The score plot is a projection of data onto subspace. It is used for interpreting relations among observations. The values 


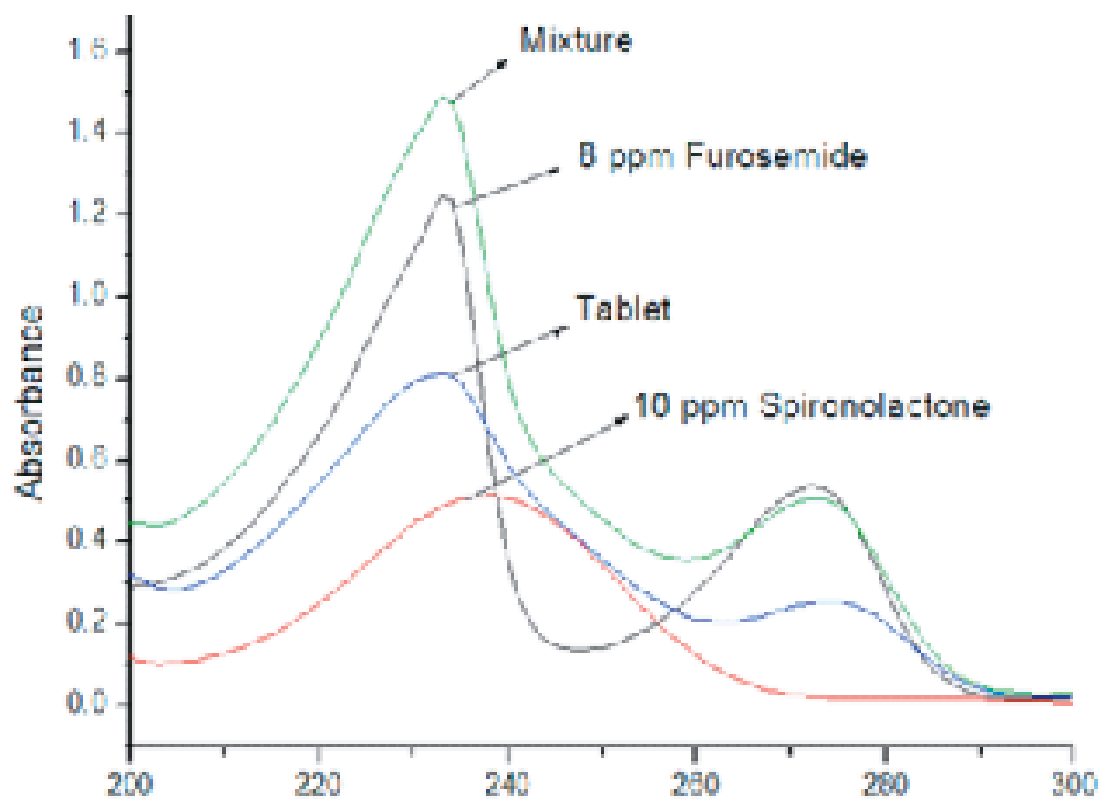

Fig. 1. Absorption spectra of $8 \mathrm{ppm}$ furosemide (at $272 \mathrm{~nm}$ ), $10 \mathrm{ppm}$ spironolactone (at $235 \mathrm{~nm}$ ) and their mixture in methanol and commercial tablet

that the spectra have in the PC coordinate system are called scores. So the 2D graphical representation is called a score plot. Each data-point in the score plot represents a spectrum. The score plot graphs each component's calculated values in relation to the other, adjusting each value for the mean and standard deviation. The Fig. 2 shows that the PC1 express
$88 \%$ and $48 \%$ of the variation whereas PC2 express $11 \%$ and $50 \%$ of the total variation in case of furosemide. But from principal component regression (PCR) of spironolactone, the Fig. 3 shows that the PC1 express $88 \%$ and $51 \%$ of the variation whereas PC2 express $11 \%$ and $49 \%$ of the total variation.

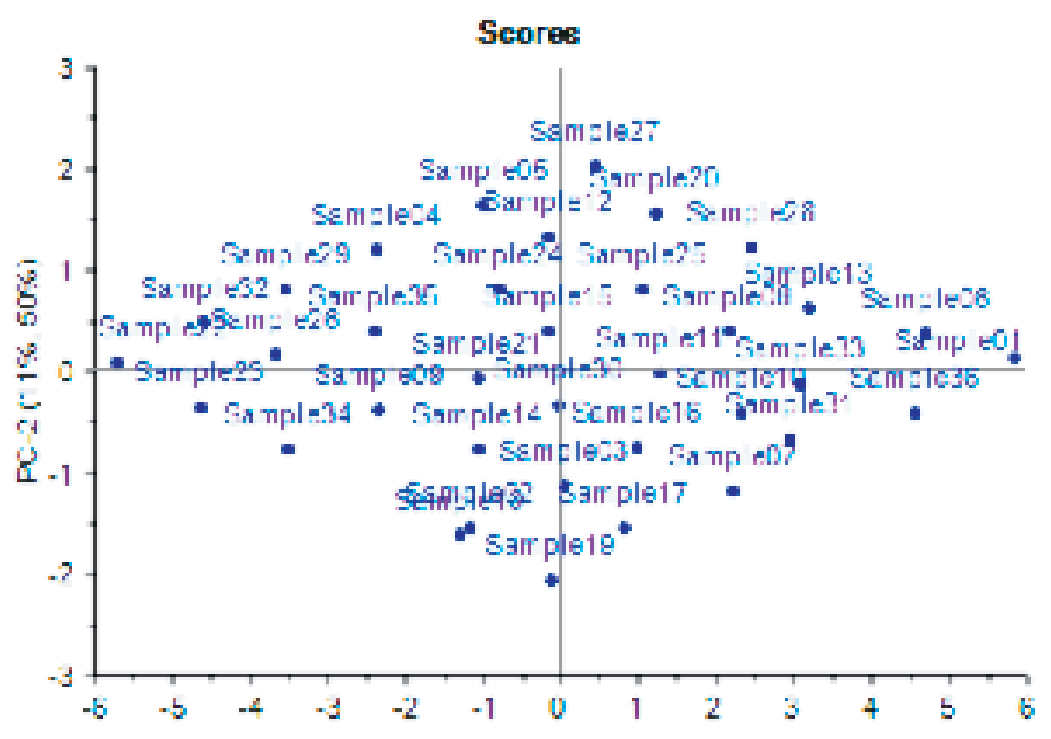

Fig. 2. Score plot of PC1 and PC2 


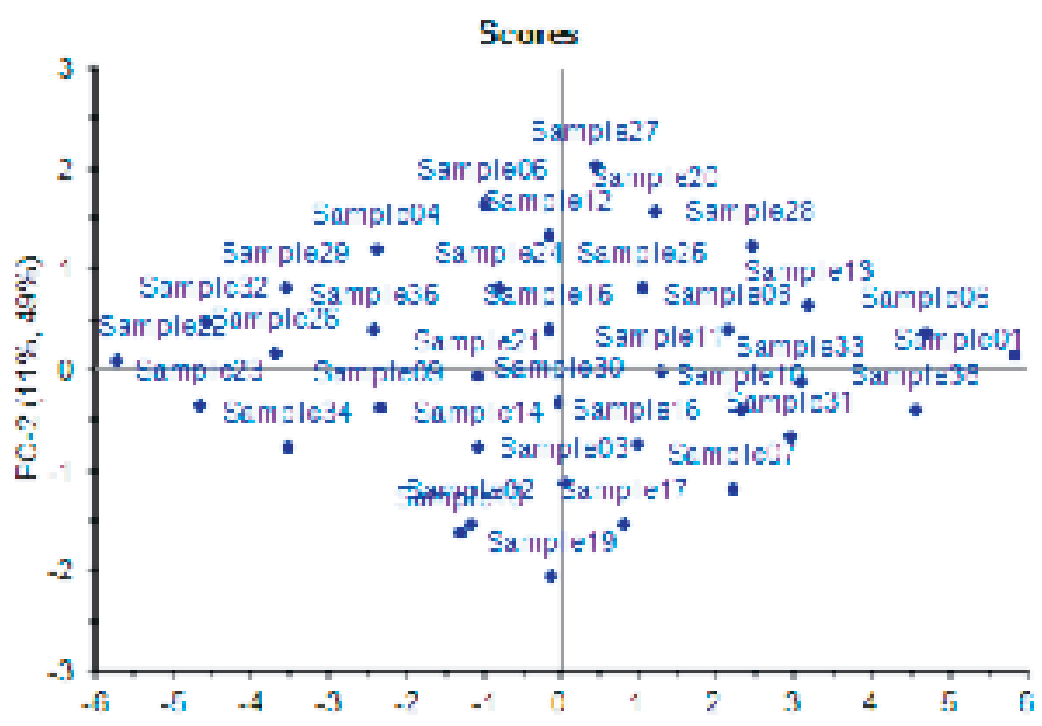

Fig. 3. Score plot of PC1 and PC2

Score plots in the above figures show that the samples are independently scattered without following any pattern.

Correlation loadings explain that some of the data are positively correlated and others are negatively correlated for both furosemide and spironolactone as shown in the following Figs. $4 \& 5$. In addition explained variance plot (figure not shown) predicts that highest explained variance is found with 2 PCs for both furosemide and spironolactone.
In Figs. $6 \& 7$ the predicted values according to PCR model are plotted against the reference values for furosemide and spironolactone respectively. The straight lines as shown in figures indicate the real values of furosemide and spironolactone that has been obtained in the analysis. But, the values obtained in the figures are indicated by points. The distribution pattern of majority data points very close to the 45 degree solid line demonstrates a good agreement between predicted and reference values.

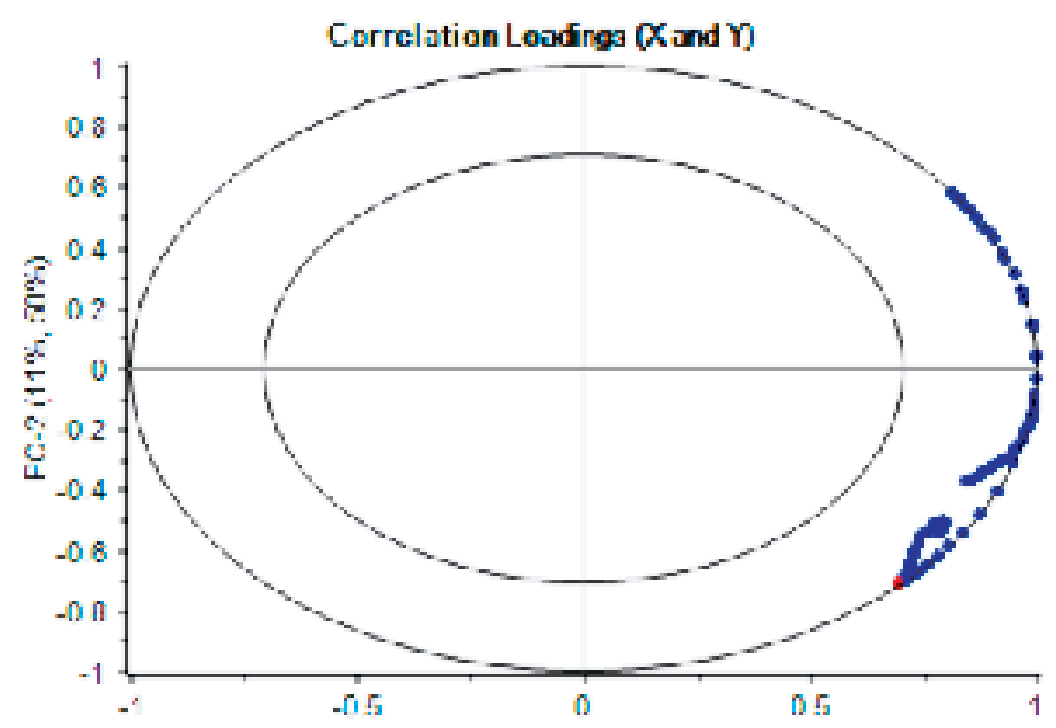

Fig. 4. Correlation loading plot (furosemide) 


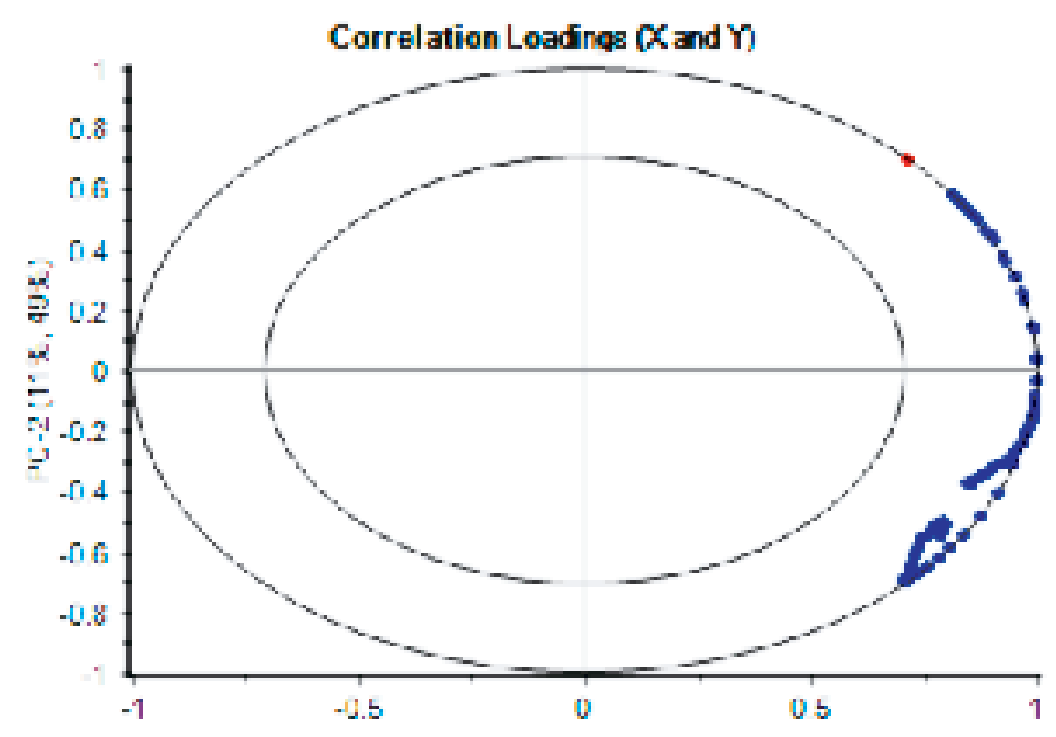

Fig. 5. Correlation loading plot (spironolactone)

\section{Application to pharmaceutical formulations}

In order to access the applicability of the proposed PCR method to the analysis of the real samples, it has been applied to the determination of these drugs in pharmaceutical formulations. Five replicate measurements for each real sample have been carried out in the present investigation. The percentage recoveries and relative standard deviations are indicated in table II. The good agreement between these results and the label claims indicate the successful applica- bility of the proposed procedures for the simultaneous determination of furosemide and spironolactone in real sample.

Mean recoveries of furosemide for PCR are found as $92.72 \%$ in Edeloss tablets, 93.44\% in Edenil tablets, 96\% in Frulac tablets, 95.05\% in Fusid Plus Tablets and 101.36\% in Resitone tablets. Similarly, mean recoveries of spironolactone for PCR are obtained as $87.52 \%$ in Edeloss tablets, $80.96 \%$ in Edenil tablets, $87.54 \%$ in Frulac tablets, $75.41 \%$ in Fusid Plus tablets and $89.94 \%$ in Resitone tablets.

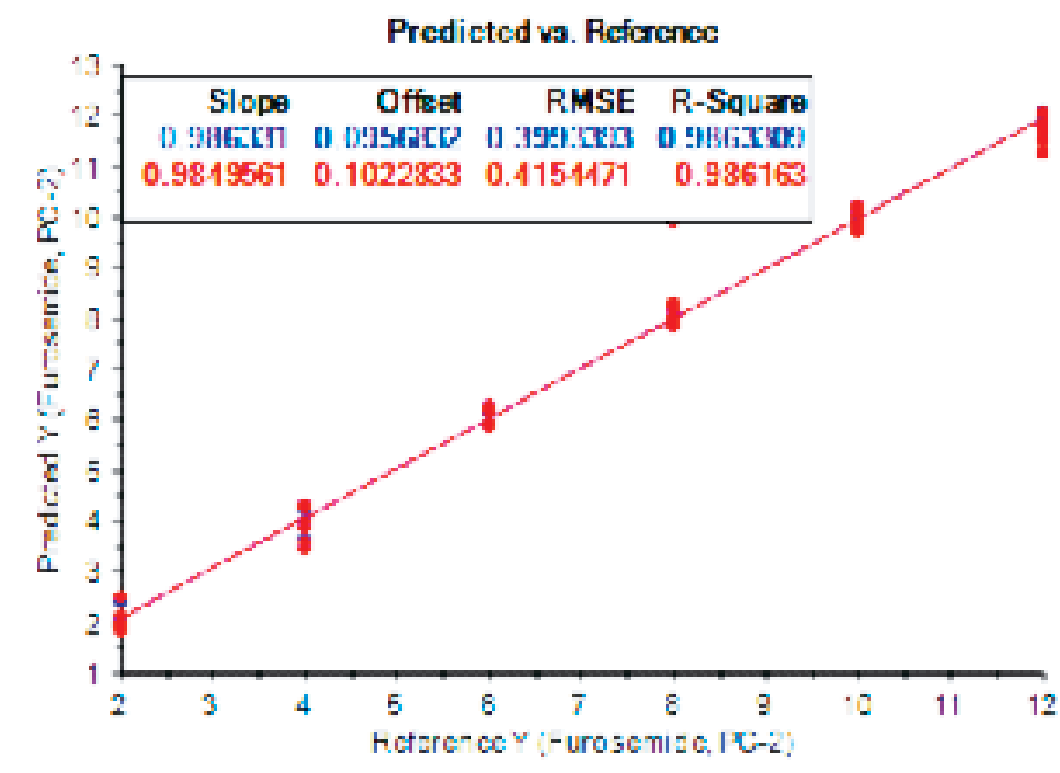

Fig. 6. Prediction vs. reference plot 
Table II. Assayed results of simultaneous determination of furosemide (furo) and spironolactone (spiro) in commercial tablets by the proposed chemometric technique

\begin{tabular}{|c|c|c|c|c|c|c|c|}
\hline \multirow[t]{2}{*}{ Manufacturer } & \multirow[t]{2}{*}{ Samples } & \multicolumn{2}{|c|}{$\begin{array}{l}\text { Label Claim } \\
\text { (mg) }\end{array}$} & \multirow{2}{*}{$\frac{\underset{\text { Furosemide }}{(\mathrm{mg})}}{\text { PCR }}$} & \multirow{2}{*}{$\begin{array}{c}\begin{array}{c}\text { Spironolactone } \\
(\mathrm{mg})\end{array} \\
\text { PCR }\end{array}$} & \multicolumn{2}{|c|}{$\begin{array}{c}\text { \% Label Claim } \\
\text { PCR }\end{array}$} \\
\hline & & Furo & Spiro & & & Furo & Spiro \\
\hline \multirow[t]{7}{*}{ Incepta Pharmaceuticals } & Edeloss 1 & & & 17.63 & 41.41 & 88.15 & 82.82 \\
\hline & Edeloss 2 & & & 17.54 & 41.04 & 87.7 & 82.08 \\
\hline & Edeloss 3 & 20 & 50 & 19.88 & 45.73 & 99.4 & 91.46 \\
\hline & Edeloss 4 & & & 19.45 & 45.13 & 97.25 & 90.26 \\
\hline & Edeloss 5 & & & 19.42 & 45.49 & 91.1 & 90.98 \\
\hline & Mean & & & 18.78 & 43.76 & 92.72 & 87.52 \\
\hline & $\mathrm{SD}$ & & & 1.11 & 2.32 & 5.53 & 4.66 \\
\hline \multirow[t]{7}{*}{ Eskayef Bangladesh Ltd } & Edenil 1 & & & 19.27 & 40.36 & 96.35 & 80.72 \\
\hline & Edenil 2 & & & 18.89 & 40.27 & 94.45 & 80.54 \\
\hline & Edenil 3 & 20 & 50 & 18.39 & 40.44 & 91.95 & 80.88 \\
\hline & Edenil 4 & & & 18.42 & 40.67 & 92.1 & 81.34 \\
\hline & Edenil 5 & & & 18.47 & 40.66 & 92.35 & 81.32 \\
\hline & Mean & & & 18.69 & 40.48 & 93.44 & 80.96 \\
\hline & $\mathrm{SD}$ & & & 0.38 & 0.18 & 1.91 & 0.36 \\
\hline \multirow[t]{7}{*}{ Orion Pharma } & Frulac 1 & & & 19.03 & 42.89 & 95.15 & 85.78 \\
\hline & Frulac 2 & & & 18.95 & 43.22 & 94.75 & 86.44 \\
\hline & Frulac 3 & 20 & 50 & 19.31 & 43.68 & 96.55 & 87.36 \\
\hline & Frulac 4 & & & 19.29 & 44.59 & 96.45 & 89.18 \\
\hline & Frulac 5 & & & 19.42 & 44.47 & 97.1 & 88.94 \\
\hline & Mean & & & 19.20 & 43.77 & 96.00 & 87.54 \\
\hline & $\mathrm{SD}$ & & & 0.20 & 0.75 & 1.00 & 1.50 \\
\hline \multirow[t]{7}{*}{ Square Pharmaceuticals Ltd } & Fusid Plus 1 & & & 20.82 & 39.88 & 104.1 & 79.76 \\
\hline & Fusid Plus 2 & & & 20.34 & 38.99 & 101.7 & 77.98 \\
\hline & Fusid Plus 3 & 20 & 50 & 19.82 & 38.13 & 99.1 & 76.26 \\
\hline & Fusid Plus 4 & & & 17.23 & 35.93 & 86.15 & 71.86 \\
\hline & Fusid Plus 5 & & & 16.84 & 35.59 & 84.2 & 71.18 \\
\hline & Mean & & & 19.01 & 37.70 & 95.05 & 75.41 \\
\hline & SD & & & 1.84 & 1.88 & 9.21 & 3.77 \\
\hline \multirow[t]{7}{*}{ Beximco Pharma } & Resitone 1 & & & 18.35 & 40.54 & 91.75 & 81.08 \\
\hline & Resitone 2 & & & 18.29 & 40.5 & 91.45 & 81 \\
\hline & Resitone 3 & 20 & 50 & 18.52 & 40.31 & 92.6 & 80.62 \\
\hline & Resitone 4 & & & 23.19 & 52.00 & 115.95 & 104 \\
\hline & Resitone 5 & & & 23.01 & 51.51 & 115.05 & 103.02 \\
\hline & Mean & & & 20.27 & 44.97 & 101.36 & 89.94 \\
\hline & SD & & & 2.58 & 6.19 & 12.92 & 12.39 \\
\hline
\end{tabular}

SD- Standard Deviation 


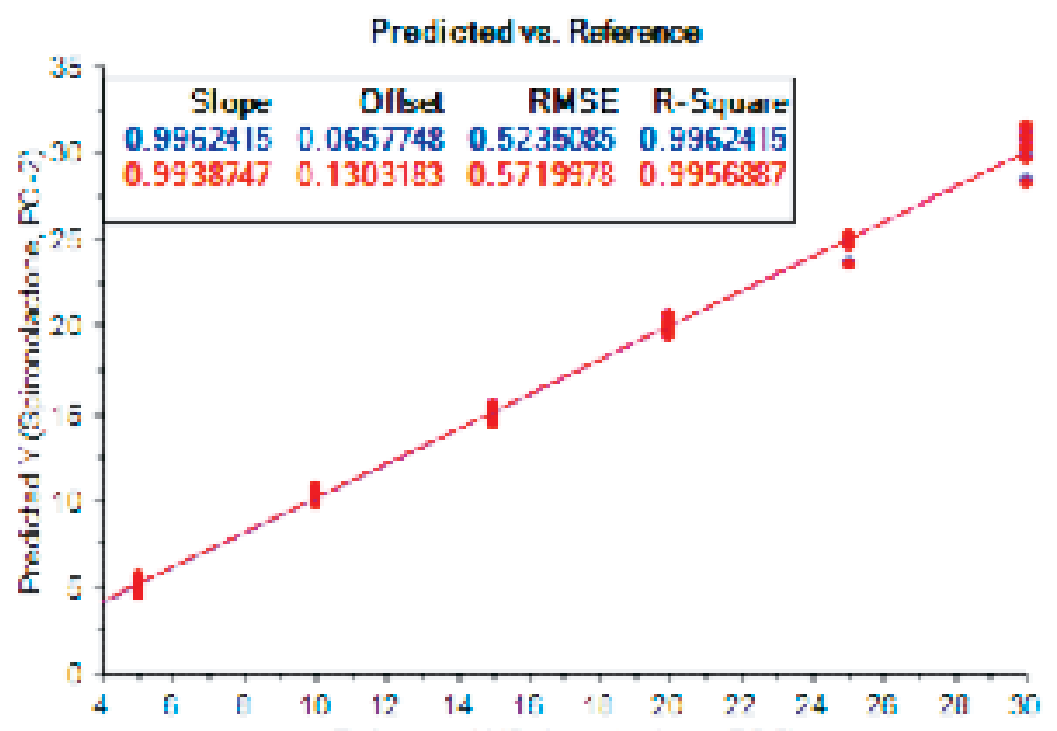

Fig. 7. Prediction vs. reference plot

Again, in order to measure the performance of PCR several tools are used. Root mean squared error (RMSE) for prediction and coefficient of determination $\left(\mathrm{R}^{2}\right)$ for prediction and classification are very much popular in statistics.

The tools for evaluating the model for prediction of furosemide and spironolactone and the prediction efficiencies are shown in the table III. In order to develop the model, $75 \%$ samples have been used for calibration and the rest are used for validation. From the table III it is obvious that prediction and validation results are significant for both furosemide and spironolactone.

Table III. Prediction efficiencies of PCR for furosemide and spironolactone

\begin{tabular}{llcc}
\hline Sample & \multicolumn{2}{c}{ PCR } \\
\cline { 3 - 4 } & & RMSE & $\mathrm{R}^{2}$ \\
\hline Furosemide & Prediction & 0.3993393 & 0.9863309 \\
& Validation & 0.4173273 & 0.9875642 \\
Spironolactone & Prediction & 0.3993393 & 0.9863309 \\
& Validation & 0.4117160 & 0.9878666 \\
\hline
\end{tabular}

\section{Conclusion}

A convenient method by using electronic absorption measurements in conjunction with PCR multivariate calibration analysis has been developed for the simultaneous determination of furosemide and spironolactone in pharmaceutical tablets. The proposed model has been evaluated by coeffi- cient of determination $\left(\mathrm{R}^{2}\right)$ and root mean squared error (RMSE). The method needs simple instrumentation, offers high sample throughput and avoids time-consuming separations or complex sample treatments, while providing a good accuracy and precision regardless of the presence of small amounts of different absorbing excipients. The results obtained in this paper demonstrate once again the capabilities of principal component regression (PCR) and encourage its application for quality control or routine analysis of pharmaceuticals.

\section{Acknowledgement}

The authors acknowledge the technical supports provided by the department of Applied Chemistry and Chemical Engineering, University of Dhaka, Materials Science \& Nanotechnology, Centre for Advanced Research in Sciences (CARS), University of Dhaka and Bangladesh Council of Scientific and Industrial Research (BCSIR), Dhaka-1205, Bangladesh.

\section{References}

Basavaiah K and Chandrashekar U (2005), Sensitive micro analysis of frusemide (furosemide) in bulk drug and formulations by visible spectrophotometry and high performance liquid chromatography (HPLC), Indian J. Chem. Techno. 12(4): 401-406. 
Brereton (2003), Chemometrics, data analysis for the laboratory and chemical plant, J. Wiley and Sons, Chichester, UK.

Beebe KR, Pell RJ and Seasholtz MB (1998), Chemometrics: a practical guide, Wiley-Interscience, UK.

Fearn (2001), Standardisation and calibration transfer for near infrared instruments: a review, J. Near Infrared Spectrosc. 9 (4): 229-244.

Ginès P, Kamath PS and Arroyo V (2010), Chronic liver failure: mechanisms and management, Springer Science \& Business Media, New York, USA.

Gole Cure A (2006), Spectrophotometric determination of furosemide in pharmaceutical dosage forms using complex formation with $\mathrm{Cu}(\mathrm{II})$, J. Anal. Chem. 61(8): 748-754.

Geladi P (2003), Chemometrics in spectroscopy. Part 1. Classical chemometrics, Spectrochim. Acta Part B 58: 767-782.

Haaland DM, Chambers WB, Keenan MR and Melgaard DK (2000), Multi-Window Classical Least-Squares Multivariate Calibration Methods for Quantitative ICP-AES Analyses, Appl. Spectrosc. 54(9): 12911302.

Keithley RB, Heien ML and Mark Wightman R (2009), Multivariate concentration determination using principal component regression with residual analysis, Trends Anal. Chem. 28(9): 1127-1136.

Lavine B (2000), Chemometrics, Anal. Chem. 72: 91-98.

Massart D, Vandeginste B, Buydens L, De Jong S, Lewi PJ and Smeyers-Verbeke J (1998), Handbook of chemometrics and qualimetrics, Elsevier, Amsterdam.

Martens H and Naes T (1991), Multivariate Calibration, J. Wiley and Sons, Chichester, UK.

Overdiek JWPM, Hermens WAJJ and Merkus FWHM (1985), Determination of the serum concentration of spironolactone and its metabolites by high-performance liquid chromatography, J. Chrom. Biomed. Sci. Appl. 341(2): 279-285.
Patel H and Solanki S (2012), Development and validation of spectrophotometric method for simultaneous estimation of furosemide and spironolactone in combined tablet dosage form, Int. J. Pharm. Pharm. Sci. 4(4): 383-386.

Parimoo P, Bharathi A and Padma K (1995), Simultaneous determinations of spironolactone with hydroflumethiazide and spironolactone with frusemide in combination formulations by UV absorption method, Indian J. Pharm. Sci. 57(3): 126-129.

Ram VR, Dave PN and Joshi HS (2012), Development and validation of a stability-indicating HPLC assay method for simultaneous determination of spironolactone and furosemide in tablet formulation, $J$. Chromatogr. Sci. 00:1-6.

Ragno G, Ioele G and Risoli A (2004), Multivariate calibration techniques applied to the spectrophotometric analysis of one-to-four component systems, Anal. Chim. Acta. 512:173-180.

Shaikh B (1995), Development and validation of a liquidchromatographic method for the determination of furosemide, a diuretic, in bovine-milk, J. Agric. Food Chem. 43(8): 2117-2121.

Salem H, El-Maamli M, El-Sadek M and Kheir AA (1991), Simultaneous determination of cilazapril and hydrochlorothiazide in tablets by spectrophotometric methods, Spectrosc. Lett. 24(3): 451-470.

Vigneau E, Devaux M, Qannari M and Robert P (1997), Principal component regression, ridge regression and ridge principal component regression in spectroscopy calibration, J. Chemom. 11(3): 239-249.

Wesley-Hadzija B and Mattocks AM (1982), Thin-layer chromatographic determination of furosemide and 4chloro-5-sulfamoyl anthranilic acid in plasma and urine, J. Chrom. Biomed. Sci. Appl. 229(2): 425-432.

Received: 23 June 2016; Revised: 29 June 2016; Accepted: 28 July 2016. 